\title{
SYLVAIN BARRÉ
}

\section{Immeubles de Tits triangulaires exotiques}

Annales de la faculté des sciences de Toulouse $6^{e}$ série, tome $9, \mathrm{n}^{\circ} 4$ (2000), p. 575-603

<http://www.numdam.org/item?id=AFST_2000_6_9_4_575_0>

(C) Université Paul Sabatier, 2000, tous droits réservés.

L'accès aux archives de la revue «Annales de la faculté des sciences de Toulouse » (http://picard.ups-tlse.fr/ annales/) implique l'accord avec les conditions générales d'utilisation (http://www.numdam.org/conditions). Toute utilisation commerciale ou impression systématique est constitutive d'une infraction pénale. Toute copie ou impression de ce fichier doit contenir la présente mention de copyright.

\section{NumDam}

Article numérisé dans le cadre du programme Numérisation de documents anciens mathématiques http://www.numdam.org/ 


\title{
Immeubles de Tits triangulaires exotiques ${ }^{(*)}$
}

\author{
SYLVAIN BARRÉ (1)
}

RÉSUMÉ. - Dans cet article, il est question d'immeubles euclidiens de type $\tilde{A_{2}}$ (appelés immeubles triangulaires). Ces immeubles de dimension deux sont intimement liés aux plans projectifs. On montre comment construire très explicitement de tels complexes et par exemple comment mettre en défaut la propriété de Desargues du plan projectif à l'infini, à distance finie.

Les deux résultats principaux sont d'une part un théorème de prescription du type des boules de rayon deux (théorème 5), et d'autre part, l'existence d'un immeuble exotique qui admet un quotient compact et dont les types des 2-boules ne sont pas tous les mêmes. Ce dernier résultat montre en particulier qu'un immeuble qui admet un quotient compact peut ne pas être homogène ; ce qui constitue une différence importante par rapport aux espaces symétriques.

Abstract. - This article is about Euclidian buildings of type $\tilde{A_{2}}$ (called triangle buildings). These 2-dimensional complexes are related to projective planes. We show in details how to construct such complexes very explicitly and, in particular, how they put Desargues property of the projectif plane at infinity down, just at a finite level.

The two main results are : first the prescription theorem (you can choose freely the type of all 2-balls : theorem 5) and secondly the existence of an exotic building which admits a compact quotient such that types of all 2-balls are not all the same. This second result proves that a building which admits a compact quotient doesn't necessairly have a vertex transitive automorphism group: this is an important difference comparing with symmetric spaces.

(*) Reçu le 18 février 2000, accepté le 19 janvier 2001

(1) Université de Bretagne-Sud, BP 573, 56017 Vannes.

e-mail: Sylvain.Barre@univ-ubs.fr 


\section{Introduction}

Les immeubles sphériques de rang $\geqslant 3$ ont été tous classifiés par Tits [T5]. En rang 2, une telle classification est hors de portée. Par contre, elle vient d'être achevée par J. Tits et Müller (voir [TM]) dans le cas des immeubles qui ont de plus la propriété de Moufang. Les trois types d'immeubles euclidiens dont le bord à l'infini est un immeuble sphérique de rang 2 , sont $\tilde{A}_{2}, \tilde{C}_{2}$ et $\tilde{G}_{2}$. Nous nous intéressons à ceux de type $\tilde{A}_{2}$, appelés immeubles triangulaires. Ils sont étroitement liés à la géométrie projective : ce sont des complexes simpliciaux simplement connexes dont les faces sont des triangles équilatéraux, et dont les links en chacun des sommets sont des graphes d'incidence de plans projectifs. Le plus petit de ces plans contient 7 points et 7 droites ; il est associé au corps à deux éléments. Un immeuble ayant cette géométire locale sera dit d'ordre 2 . Les deux principaux résultats de cet article se placent dans ce contexte des immeubles triangulaires d'ordre 2 . En 1988, J. Tits démontrait le très joli résultat suivant [T1]:

THÉORÈme 1. - (J. Tits) Dans les immeubles triangulaires d'ordre 2, il existe exactement deux types de boules de rayon combinatoire 2 (à isomorphisme près).

Ainsi, les sommets d'un immeuble triangulaire d'ordre 2 se trouvent intrinsèquement colorés par le type de leurs 2-boules. On conviendra que les sommets blancs (resp. noirs) sont ceux de l'immeuble associé à $S L_{3}\left(\mathbb{F}_{2}((T))\right)$ (resp. $S L_{3}\left(\mathbb{Q}_{2}\right)$ ). Ce bicoloriage permet de voir se dessiner dans un immeuble triangulaire d'ordre 2 , une structure géométrique supplémentaire.

Le premier théorème principal de ce texte (théorème 5 de prescription), montre qu'il est possible de choisir en toute liberté cette coloration. Par exemple, la prescrition hexagonale dessine un pavage hexagonal dans chacun des plats : chaque hexagone est composé de six triangles, le sommet central est d'une couleur tandis que les six sommets du bord sont de l'autre. La clé de la preuve de ce théorème de prescription se trouve dans la nouvelle démonstration du théorème 1 de Tits (énoncé plus haut) que nous présentons et qui se résume sur la figure 3 . La plupart de ces prescriptions donnent naissance à des immeubles ayant un groupe d'automorphismes trivial. Tout à fait à l'opposé, se trouvent les immeubles classiques, c'est-àdire ceux associés aux groupes $S L_{3}(K)$, où $K$ est un corps local de corps résiduel à deux éléments dans le cas des immeubles triangulaires d'ordre 2. Van Steen [VS] démontre que ces immeubles classiques sont exactement ceux qui admettent une BN-paire, c'est-à-dire une action fortement transitive (i.e. transitive sur les couples chambre $\subset$ appartement). Mais entre 
les deux, il y a des immeubles qui admettent des quotients finis sans pour autant être classiques. [CMSZ] donnent de tels exemples pour l'ordre 3. Les immeubles en question admettent une action simplement transitive sur l'ensemble de leurs sommets. Aussi, pour l'ordre 2, M. A. Ronan [R3] donne des immeubles (dont on ne connaît pas encore la nature) qui admettent des quotients à trois sommets. Dans ce cas, le groupe de tous les automorphismes des immeubles en question, n'est pas déterminé exactement. L'analogie entre variétés compactes à courbure $\leqslant 0$, de rang deux et polyèdres de rang deux décrite dans [Ba1] nous invite à se demander par exemple si un immeuble qui admet un quotient compact admet nécessairement une action transitive sur l'ensemble de ses sommets (un espace symétrique est homogène), ou si les groupes d'isotropie (sous-groupes du groupe des automorphismes formés des éléments qui fixent un sommet) sont nécessairement infinis. Dans le cas classique, ceux-ci sont infinis non dénombrables. Le second résultat principal de cet article (théorèmes 6 et 7) vient répondre à ces questions par la négative en apportant un exemple d'immeuble triangulaire d'ordre 2 qui admet un quotient à deux sommets et dont les groupes d'isotropie sont d'ordre inférieur à 12. Rappelons qu'il n'y a pas d'exemple d'immeuble exotique qui admette des quotients à un seul sommet (voir [Ba2] et [CMSZ]). L'intérêt de notre exemple, comparé à ceux de Ronan, provient surtout du fait que ses deux sommets n'ont pas même couleur dans l'immeuble (théorème 6). Et c'est la structure géométrique ainsi déterminée intrinsèquement dans l'immeuble qui fait apparaître de façon extrêmement limpide que le groupe d'isotropie est fini. Bien sûr, on pourrait se demander si l'exemple que nous fournissons ne fait pas partie de la liste de ceux de Ronan, mais il n'en est rien car notre exemple n'admet pas de quotient à trois sommets (il y a autant de sommets d'un type que de l'autre). Par contre, il n'est pas exclu que les exemples de Ronan réalisent une prescription hexagonale (dans le sens du théorème 5). Cela mériterait d'être précisé. Mais cette prescription serait trop symétrique pour, à elle seule, imposer un groupe d'isotropie fini.

Dans une première partie, nous avons voulu donner une construction générale des immeubles triangulaires (d'ordre quelconque). Cette partie ne contient pas de résultats nouveaux importants, mais elle met en place un certain langage, et introduit de nombreux dessins et des techniques de construction qui sont essentielles pour la suite. Dans la seconde partie, on donne une preuve du théorème de prescription. Enfin, la troisième partie présente un immeuble exotique et montre ses propriétés particulières, ce qui compose le second théorème principal de cet article. 


\section{La tour des links}

Dans cette partie, $\mathrm{X}$ désigne un immeuble triangulaire d'ordre quelconque $q$. La plupart des figures sont réalisées dans le cas $q=2$, mais elles illustrent bien, sans perte d'information, le cas général. Nous commençons par montrer à quoi ressemblent les boules de rayon 2 de $X$. En particulier, on donnera la preuve du théorème 1 de Tits, rappelé dans l'introduction.

\subsection{Le link d'ordre 2}

Fixons-nous un sommet $S \operatorname{de} X$. Le link $L_{1}$ en ce sommet (ou link d'ordre un) s'identifie à la sphère de rayon combinatoire un (ou à la sphère métrique de rayon $\frac{1}{2}$ en supposant que les arêtes sont de longueur un). Regardons maintenant la sphère de rayon $\frac{3}{2}$. Ce sera cette sphère que nous appellerons link d'ordre 2 au sommet $S$, on le note $L_{2}$. Les longueurs des arêtes de ce link ne nous intéressent pas. On peut voir $L_{2}$ au-dessus de $L_{1}$ sachant que $X$ est étoilé sur $S$. Plus précisément, on dira qu'au-dessus d'une arête se trouvent celles correspondant aux faces la contenant, et qu'au-dessus d'un sommet se trouvent les autres arêtes intervenant dans le link en ce sommet. De plus, on dira qu'un sommet $H$ est au-dessus d'un sommet $B$ si $H$ se trouve sur un rayon issu du centre $S$ et passant par $B$. Dans le cas $q=2$, la figure 1 montre le voisinage de l'arête $a$.
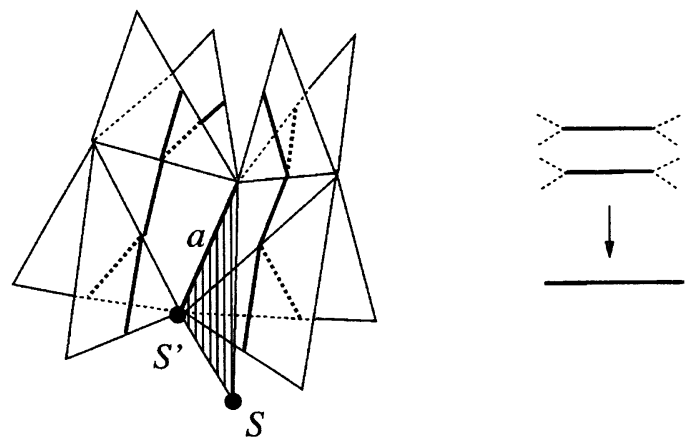

Figure 1

Construisons $L_{2}$ à partir de $L_{1}$. Pour cela commençons par le cas le plus simple $(q=2)$. On rappelle qu'alors le link est le graphe d'incidence du plan projectif $P^{2}\left(\mathbb{F}_{2}\right)$.

Six «arêtes de poisson» viennent se recoller au-dessus d'un sommet de $L_{1}$. La figure 1 montre comment une arête (notée $a$ ) du link donne naissance 
à deux arêtes de poisson qui sont les arêtes au-dessus d'elle avec à leurs extrémités, les arêtes voisines, appelées fourches et notées en pointillés sur la partie de droite de la figure 1.
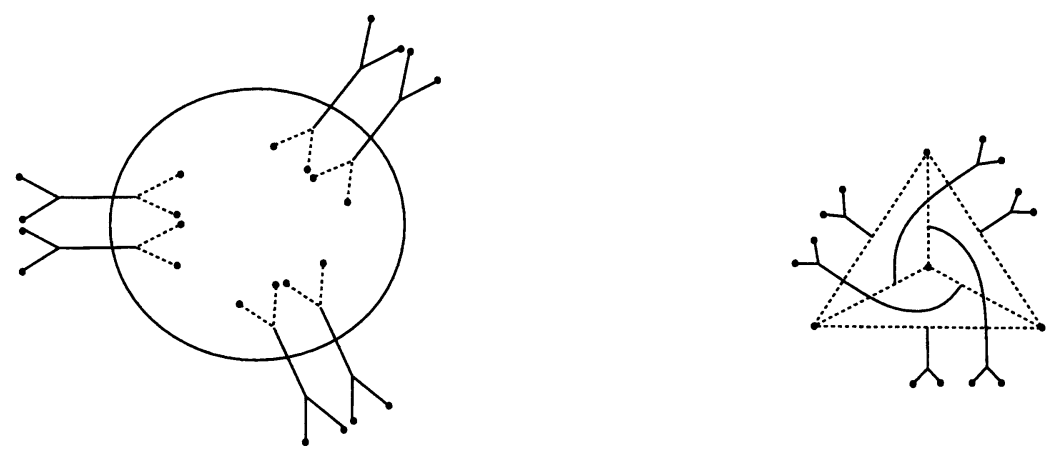

Figure 2

Pour mieux comprendre ce recollement, nous allons regarder l'ensemble des fourches des arêtes pour voir le graphe qu'elles forment (voir figure 2). Sur la figure 1, regardons le link au sommet $S^{\prime}$. L'arête $S S^{\prime}$ dans ce link correspond à un sommet que nous penserons comme une droite à l'infini. Le dessin s'éclaire alors : les six fourches correspondent aux six droites restantes du plan projectif quand on lui a enlevé la droite à l'infini. Elles vont par paires de droites parallèles. L'ensemble cherché est donc un plan affine. Dans le cas $q=2$, on peut y penser comme à un tétraèdre : les points sont les sommets et les droites les milieux des arêtes (deuxième partie de la figure 2). Deux droites parallèles sont deux droites opposées dans le tétraèdre.

Ainsi donc se construit un candidat au link d'ordre deux $L_{2}$ au-dessus du link $L_{1}$ :

1. Au-dessus de chaque arête, placer $q$ arêtes,

2. Au-dessus de chaque sommet, placer un plan affine,

3. Relier les arêtes aux plans affines vers lesquels elles se dirigent, en respectant le parallélisme.

Dans le cas $q=2$, cela donne la figure 3 .

Nous sommes maintenant en mesure de démontrer le théorème 1 de Tits.

Démonstration. - Observons le «shadok » dessiné à droite de la figure 2. L'action d'un demi-tour sur le tétraèdre correspond à la permutation simultanée de deux des trois directions. Considérons maintenant un dessin dans le plan d'un candidat au link d'ordre deux. La symétrie que nous venons de mettre en évidence nous informe qu'on peut supposer qu'autour d'un même 
shadok, il y a au plus une seule direction vrillée. Ces brins vrillés, c'est-à-dire qui se croisent, se trouvent alors isolés. Prenons-en un et rejoignons-le abstraitement au plus proche par un plus court chemin (dans $L_{1}$ ). En opérant des demi-tours sur les tétraèdres à l'intérieur du chemin, on fait glisser une vrille jusqu'à ce qu'elle rejoigne l'autre et alors ces deux dernières vrilles se détruisent ensemble. Ainsi, il apparait au plus deux classes d'isomorphismes de link suivant qu'il reste ou non un brin vrillé après ces opérations de destructions mutuelles. Bien sûr, il reste à voir pourquoi le link non vrillé et le link une fois vrillé ne sont pas isomorphes. Il suffit pour cela de constater qu'un automorphisme de $L_{2}$ est toujours au-dessus d'un automorphisme de $L_{1}$. L'ensemble des petits cycles de longueur 6 qui correspondent aux tétraèdres est préservé. Ainsi un automorphisme envoie tétraèdre sur tétraèdre. Pour conclure, il faudrait voir que ces deux candidats au link d'ordre 2 apparaissent effectivement dans un immeuble triangulaire. On verra plus loin que tous ces links sont réalisables.

On dira que le link d'ordre 2 une fois vrillé (resp. non vrillé) est de type 1 (resp. de type 0).

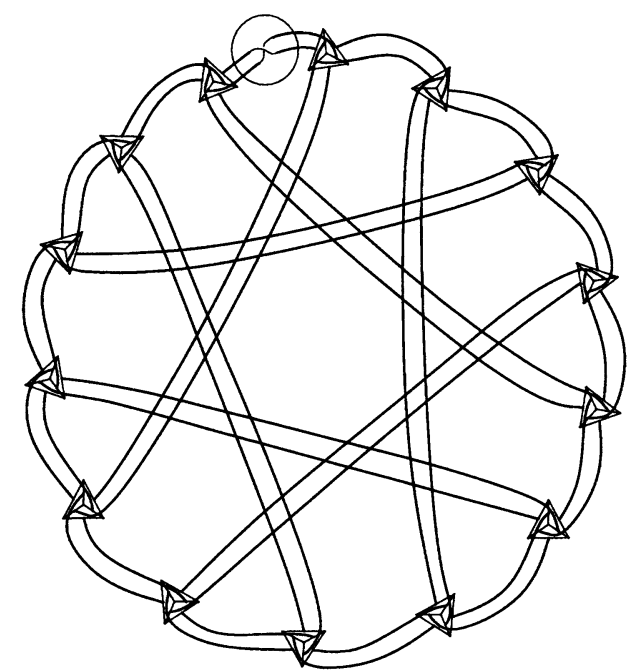

FiguRE 3

Pour $q \geqslant 3$, il y a beaucoup plus de classes d'isomorphismes (voir [T1]). Regardons simplement le cas $q=3$. Ici, les links correspondent au plan projectif sur le corps à trois éléments (c'est le seul link possible pour $q=3$ ). Les isomorphismes de l'espace affine au-dessus d'un sommet sont engendrés par les translations et les homothéties. Les premières fixent une direction et permutent cycliquement les trois autres ; les secondes permutent deux des trois brins de chaque direction. (voir la figure 4) 


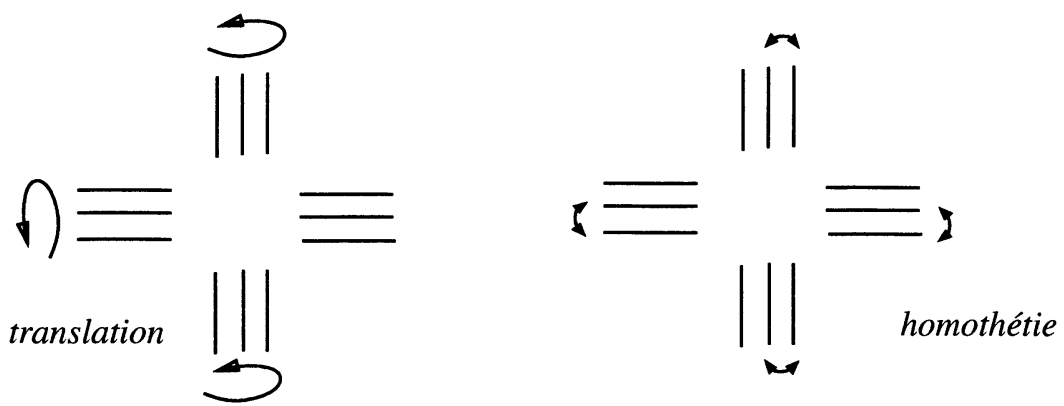

FIGURE 4

Une droite affine d'au moins 4 points possède moins de transformations affines que de bijections $(q(q-1)<q$ !). Le cas $q=4$ est un peu spécial car le corps à quatre éléments possède un automorphisme d'ordre deux et ainsi toutes les bijections sont projectives. Mais pour $q \geqslant 5$, il apparaît des links d'ordre 2 qui ne peuvent pas être links d'immeubles classiques. Expliquons cela plus en détail.

Soit $a$ une arête reliant deux sommets $S_{1}$ et $S_{2}$. Cette arête correspond dans le link $L_{1}$ (resp. $L_{2}$ ) en $S_{1}$ (resp. $S_{2}$ ) à une droite projective $D_{1}$ (resp. $D_{2}$ ). Les faces contenant l'arête $a$ déterminent une application $\varphi: D_{1} \longrightarrow$ $D_{2}$. Tout automorphisme $\Psi_{2}$ de $L_{2}$ qui fixe $D_{2}$ globalement (i.e. le sommet correspondant à l'arête $a$ dans le link $L_{2}$ ) opère donc sur $D_{2}$. On peut maintenant donner la définition suivante :

DÉfINITION 1.1. - On dira qu'un immeuble est régulier si pour toute arête $a$ et tout automorphisme $\Psi_{2}$ comme ci-dessus, l'action sur $D_{1}$, conjuguée par $\varphi$ de l'action de $\Psi_{2}$ sur $D_{2}$, provient de la restriction d'un automorphisme de $L_{1}$ qui fixe $D_{1}$ globalement.

Ainsi, dans un immeuble régulier, à une arête correspond une droite projective. Notons bien sûr, qu'un immeuble classique est régulier. L'existence d'immeubles non classiques devient alors claire grâce à cette notion. En effet, il suffit de permuter les brins suivant une transformation qui n'est pas une homographie pour obtenir un link d'ordre deux qui ne peut être classique.

\subsection{Construction de la tour des links}

Nous allons maintenant décrire les links d'ordre supérieur dans $X$. Pour passer aux links d'ordre 3 et plus, il y a une petite difficulté qui provient du fait suivant : 
Pour la sphère $S_{1}$, tous les sommets sont équivalents. Par contre, dans $S_{2}$ et, plus généralement, dans la sphère combinatoire $S_{n}$ de rayon $n$ pour $n \geqslant 2$, il apparaît deux types de sommets : les coins et les autres. Les coins se projettent sur un sommet dans $S_{1}$, alors que les non coins se trouvent au-dessus d'une arête. (voir la figure 5)
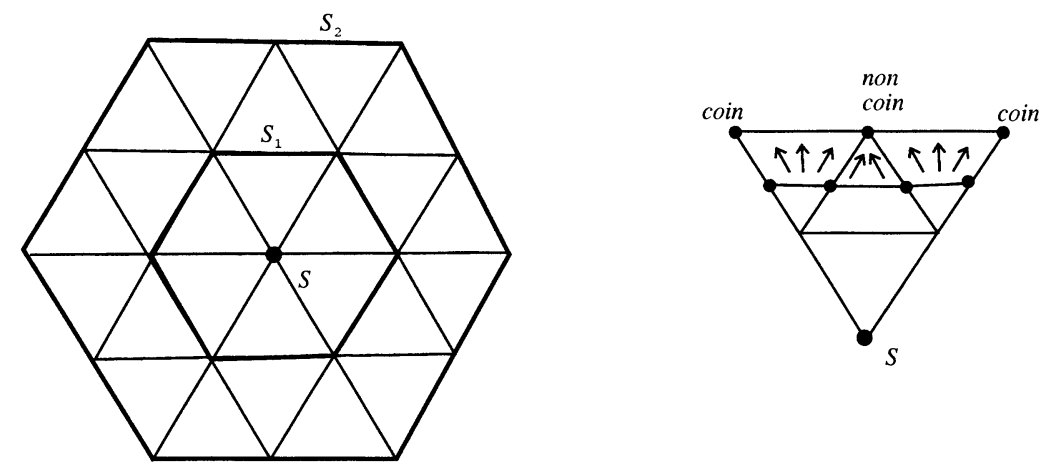

FiguRE 5

Une autre chose à remarquer est que la sphère $S_{2}$ et le link d'ordre deux $L_{2}$ ne sont pas identiques. Pour passer de $L_{2}$ à $S_{2}$, il faut écraser les arêtes $a$ qui ont été dédoublées pour former $L_{2}$ au dessus de $L_{1}$ (se référer à la figure 5).

Remarquons que les sommets de $S_{2}$ non coins auront une valence 4 dans le cas $q=2$ et $2 q$ en général. Voyons maintenant comment obtenir $L_{3}$ audessus de $S_{2}$. Ensuite, il en sera de même pour obtenir $L_{n+1}$ au-dessus de $S_{n}$ pour $n \geqslant 2$. Se référer à la figure 6 pour ce qui suit. Nous distinguons trois points :

1. Dédoubler les arêtes de $S_{2}$ (ou, en général, en mettre $q$ au-dessus de chacune),

2. Au-dessus d'un coin, placer un plan affine (comme pour former $L_{2}$ au-dessus de $L_{1}$ ),

3. Au-dessus des autres sommets (les «non coins»), ajouter le morceau de plan affine manquant (on précise cela ci-dessous).

De même qu'au niveau d'un coin, on a déjà remarqué qu'il fallait compléter un plan affine pour former un plan projectif, dans l'étape 3 , il s'agit de compléter un graphe en un plan projectif. On constate que le graphe en question est un arbre (l'arête $a$ est centrale dans cet arbre, voir figure 6) et donc qu'il se complète en un plan projectif (l'étude des immeubles métriques dans [Ba1] permet de voir cela). Dans le cas $q=2$, c'est un octogone qu'il faut ajouter, et plus précisément, la figure 7 montre comment les huit brins 
viennent se recoller : une paire sur des sommets opposés et deux paires provenant d'un même sommet sur des diagonales orthogonales.
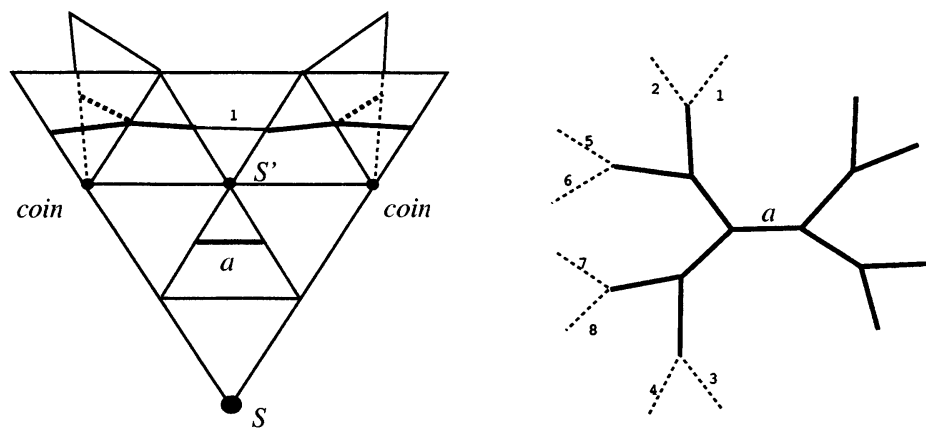

Link en $S^{\prime}$

FIGURE 6

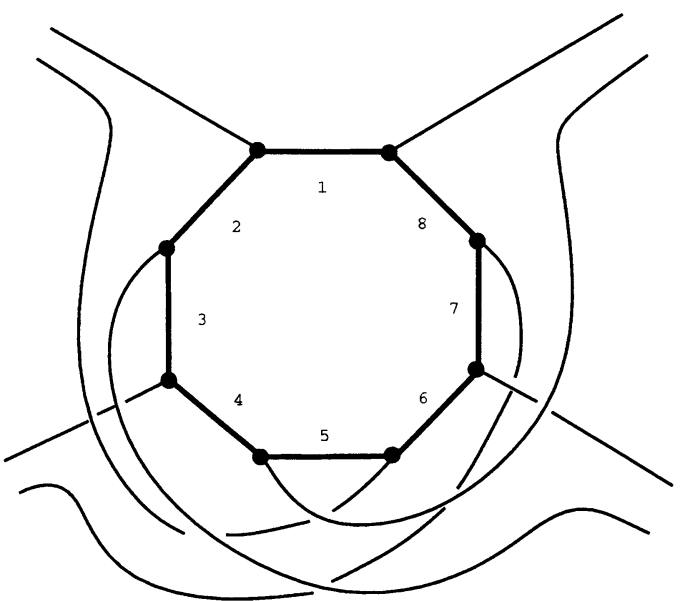

FigURE 7

En conclusion nous pouvons résumer la construction que nous venons d'expliciter par la figure schématique (figure 8).

Jusqu'ici, nous avons regardé comment étaient construits les links d'un immeuble. Maintenant, on se propose d'étudier la réciproque et donc de construire un immeuble qui repose sur une tour. Pour cela donnons la définition suivante :

DÉFINITION 1.2. - Une tour de links est une succession infinie d'éclatements et d'écrasements au-dessus d'un link de base avec les règles décrites plus haut. 


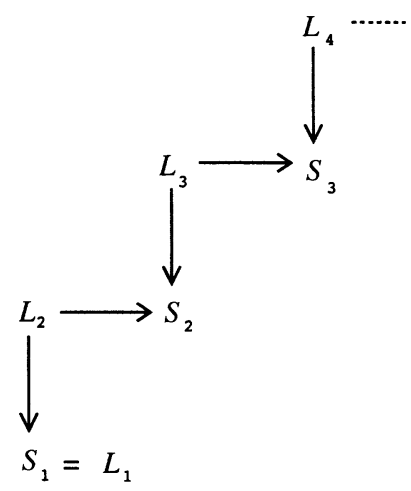

Figure 8

Le link de base est n'importe quel plan projectif et peut varier suivant les sommets. À une tour de links correspond un complexe qui vérifie le critère local des immeubles (avoir pour link le graphe d'incidence d'un plan projectif). Ici, le complexe obtenu à partir d'une tour est simplement connexe (car contractile) nous pouvons donc affirmer (d'après [T2]) qu'il s'agit d'un immeuble triangulaire. Finalement, le résultat obtenu s'énonce ainsi :

THÉORÈME 2. - À une tour de links correspond un et un seul immeuble triangulaire pointé.

Dans [R2], on trouve une construction analogue à celle-ci. Mais nous avons voulu préciser des notations et surtout introduire des figures qui seront essentielles pour ce qui suit. Il faudra en particulier toujours conserver en tête l'image de la «ronde des shadoks » (figure 3), qui prouve le théorème 1 de Tits, et celle de l'octogone (figure 7).

À la lumière de cette tour de links, on peut faire deux remarques sur ces immeubles.

Remarque 1 : Il existe des immeubles triangulaires pour $q=2$ qui ne sont pas homogènes sur les sommets étiquetés. (On peut toujours étiqueter l'ensemble des sommets d'un immeuble triangulaire à l'aide de trois couleurs, de telle sorte que chaque chambre ait trois sommets de couleurs différentes).

Vérifions cela. Donnons-nous les quatre premiers étages d'une tour centrée sur le sommet $S$. Sur le second étage, fixons-nous un point $S^{\prime}$ de même étiquette que $S$. Si son link d'ordre 2 est identique à celui de $S$, il est possi- 
ble de le modifier en permutant deux faces de la sphère de rayon 4 (voir la figure 9). Ensuite, on complète la tour d'une façon quelconque et on obtient ainsi un immeuble avec la propriété voulue. En fait, à travers l'étiquetage, nous avons voulu souligner le fait qu'il est possible de créer un immeuble, en ayant comme contrainte que deux sommets choisis aient des links d'ordre 2 différents. Nous verrons dans la seconde partie un résultat bien plus précis que celui-là.

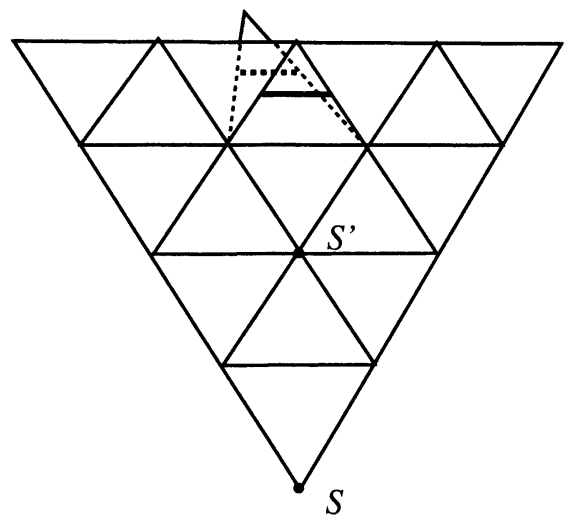

FIGURE 9

Remarque 2 : Il existe un infinité non dénombrable d'immeubles triangulaires d'ordre fixé (même dans le cas $q=2$ ).

Il y a un nombre dénombrable de sommets dans un immeuble et donc à un immeuble, on ne peut associer qu'un nombre au plus dénombrable de tours distinctes. Or il y a une infinité non dénombrable de tours (à chaque étage, on a au moins un choix à faire). Ainsi, même en restant parmi les immeubles triangulaires correspondant à $q=2$, on obtient une infinité non dénombrable d'exemples deux à deux non isomorphes. Remarquons qu'une sphère de rayon $n$ détermine par projections successives tous les étages inférieurs de la tour et qu'il y a donc au moins $2^{n}$ sphères de rayon $n$ deux à deux non isomorphes.

En conclusion de cette partie, nous pouvons noter que la construction à partir d'une tour fournit un immeuble pointé et ne maîtrise en rien l'aspect homogène. Quel lien y a-t-il entre deux tours d'un même immeuble centrées en des points différents ; comment reconnaitre l'homogénéité? Voilà deux questions qu'on n'aborde pas par cette construction.

Par contre, nous avons obtenu des moyens simples pour construire des immeubles localement symétriques (i.e. dont les links sont des plans pro- 
jectifs classiques) mais pas symétriques (i.e. il y a des isométries locales qui ne proviennent pas d'isométries globales) car pas réguliers ; ou bien, pour construire des immeubles non homogènes. De plus, en particulier dans le cas $q=2$, nous pouvons théoriquement distinguer deux immeubles homogènes (par exemple classiques) en comparant leurs tours. Nous verrons dans le paragraphe suivant que le link d'ordre trois pourra faire apparaitre le caractère non classique dans le cas $q=2$.

\subsection{La propriété de Desargues du bord à l'infini vue à distance finie}

Le bord à l'infini d'un immeuble triangulaire, comme ses links, est un plan projectif. Rappelons qu'un plan projectif qui vérifie la propriété de Desargues est le plan projectif sur un corps $[\mathrm{A}]$. On se propose ici de vérifier cette propriété de Desargues du bord à l'infini, à distance finie, à travers les sphères $S_{n}$ de rayon $n$. Cela nous donnera un critère simple qui assure du caractère non classique d'un immeuble. Dans [VM2], Van Maldeghem a donné une interprétation des sphères de rayon $n$. Il s'agit de plans de Hjelmslev. Nous ne donnons pas ici d'interprétation générale de ce type, mais, suite aux constructions très précises que nous avons données plus haut, nous précisons encore comment reconnaitre la propriété de Desargues.

\subsubsection{Points et droites de $S_{n}$}

Fixons un sommet de $X$ et regardons la tour des links en ce sommet.

DÉFINITION 1.3. - Dans le graphe $S_{n}$, on appellera points (resp. droites) les sommets qui se projettent sur des points (resp. droites) dans $S_{1}$. Les autres sommets sont sans nom.

Avec cette définition, on conviendra qu'un point est sur une droite s'il y a un chemin dans $S_{n}$ de l'un à l'autre, ne rencontrant que des sommets sans nom.

Remarque. Par deux points de $S_{n}$, qui se projettent sur deux points distincts dans $S_{1}$, passe une et une seule droite. Par contre, par deux points au-dessus d'un seul dans $S_{1}$ passent plusieurs droites. Par exemple, dans le cas $q=2$, par deux tels points de $S_{2}$, passent exactement deux droites (voir la construction de la tour des links).

\subsubsection{Propriété de Desargues au niveau $n$}

Pour commencer, rappelons la propriété de Desargues dans un plan projectif. 
DÉFINITION 1.4. - Une configuration de Desargues dans un plan projectif est la donnée de deux vrais triangles $(A B C)$ et $\left(A^{\prime} B^{\prime} C^{\prime}\right)$ en perspective : c'est-à-dire tels que les droites $\left(A A^{\prime}\right) .\left(B B^{\prime}\right)$ et $\left(C C^{\prime}\right)$ (appelées fuyantes) soient concourantes.

DÉfINITION 1.5. - On dit qu'un plan projectif vérifie la propriété de Desargues si pour toute configuration de Desargues $(A B C)$ et $\left(A^{\prime} B^{\prime} C^{\prime}\right)$, les trois points $(A B) \cap\left(A^{\prime} B^{\prime}\right),(A C) \cap\left(A^{\prime} C^{\prime}\right)$ et $(B C) \cap\left(B^{\prime} C^{\prime}\right)$ sont alignés.

Nous pouvons illustrer cette propriété par la très célèbre figure suivante :

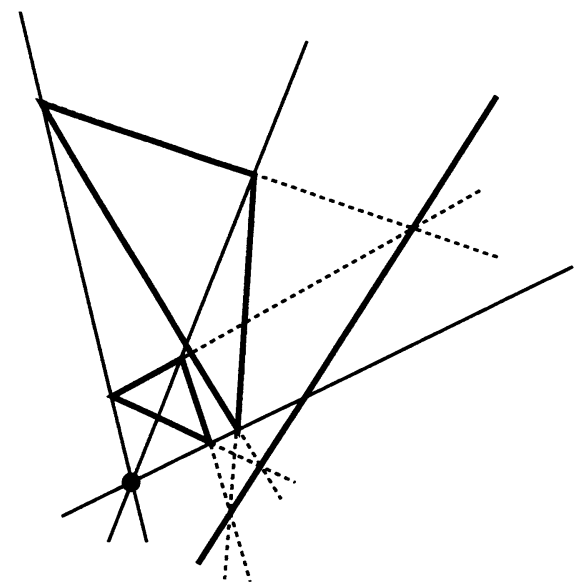

Figure 10

Ces définitions étant précisées, nous pouvons rappeler le théorème classique suivant.

THÉORÈME 3. - Un plan projectif $P$ vérifie la propriété de Desargues si et seulement si il existe un corps $K$ tel que $P=P^{2}(K)$.

Le caractère nécessaire de cette propriété est un exercice bien connu. Voir $[\mathrm{A}]$ pour la démonstration de la réciproque.

Dans le cas de la sphère $S_{n}$, entendons la propriété de Desargues en ce sens : pour toute configuration de Desargues qui est la projection d'une configuration de Desargues à l'infini, les projetés des trois points d'intersections de la configuration à l'infini sont sur une même droite.

Nous pouvons maintenant énoncer le fait qui nous a conduit à toutes ces définitions. 
Proposition 1.1. - Le plan projectif à l'infini vérifie la propriété de Desargues si et seulement si cette propriété est vérifiée par toutes les sphères $S_{n}$.

Démonstration. - On rappelle que nous avons pointé un immeuble une fois pour toutes. Dans cet énoncé, une implication est tautologique. Supposons donc la propriété de Desargues vérifiée à tous les niveaux finis. Considérons une configuration de Desargues à l'infini. Dans chacune des sphères $S_{n}$, nous obtenons par hypothèse une ou plusieurs droites qui contiennent les trois projections des trois points d'intersection dont on veut démontrer qu'ils sont alignés à l'infini. Notons $\mathcal{D}_{n}$ l'ensemble des droites solutions au niveau de $S_{n}$. Remarquons que $\mathcal{D}_{n+1}$ se projette naturellement sur $\mathcal{D}_{n}$. Ainsi, il existe donc une suite $\left(D_{n}\right)$ de droites telles que $D_{n} \in \mathcal{D}_{n}$ et $D_{n+1}$ se projette sur $D_{n}$. Cette suite détermine à l'infini une droite qui répond à la question.

En pratique, il semble difficile de vouloir montrer le caractère classique du bord à l'infini, en regardant les sphères de rayon $n$. Pourtant, c'est ce qu'est parvenu à faire Van Steen pour prouver que les immeubles triangulaires classiques sont ceux associés à des BN-paires. Par contre, il sera très facile de démontrer le caractère non classique en exhibant une sphère $S_{n}$ qui ne l'est pas.

\subsubsection{Des sphères non classiques}

Les cas $q=2$ et $q \geqslant 3$ se distinguent ici. Dans le second cas, il est possible de nier la propriété de Desargues dès le niveau 2 alors que dans le premier on ne peut le faire qu'à partir du niveau 3. De plus il est possible de faire cela tout en conservant des liaisons projectives. En d'autres termes, un immeuble peut être régulier sans pour autant vérifier la propriété de Desargues. C'est peut-être dans cette dichotomie (à partir du niveau 2 ou bien à partir du niveau 3 seulement) que réside la raison profonde du fait que [CMSZ] trouvent des groupes qui opèrent simplement transitivement sur les sommets d'immeubles non classiques dans le cas $q=3$ et pas dans le cas $q=2$.

\section{Le cas $q \geqslant 3$.}

Afin d'avoir une image en termes de relations d'incidence d'une configuration de Desargues, voici un graphe (figure 11) qui correspond au cas le plus général. Au centre, on trouve le point de concours des trois fuyantes. Si la propriété de Desargues est vérifiée, la figure se complète symétriquement (par les traits pointillés). Dès que $q \geqslant 3$, il est possible de trouver une telle 
configuration. Sur la figure, les points noirs correspondent à des points et les blancs à des droites.

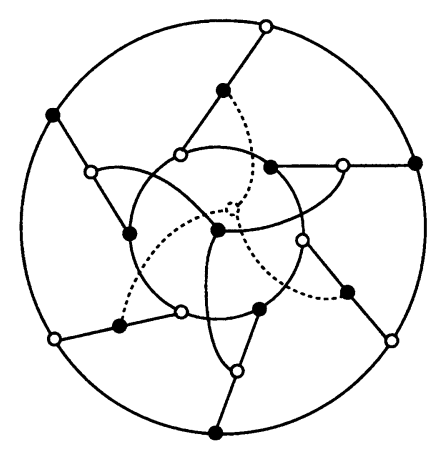

Figure 11

Fixons-nous un link (classique par exemple) avec $q \geqslant 3$. Nous allons voir qu'il est possible de construire un link d'ordre 2 au-dessus du link en question, qui ne vérifie pas la propriété de Desargues. Il faut ici mettre une chose au clair. La définition que nous avons donnée de cette propriété au niveau $S_{n}$ dépend de l'immeuble tout entier ; cependant, on peut trouver un link d'ordre 2 qui ne pourra vérifier cette propriété quel que soit l'immeuble dans lequel il est placé. Pour cela, vérifions qu'une configuration de Desargues générique (comme celle qui est dessinée plus haut, c'est-à-dire où tous les points et droites considérés sont deux à deux disjoints), provient toujours d'une configuration de Desargues à l'infini. Nous allons nous assurer que le relèvement à un étage immédiatement supérieur est possible.

Le lemme suivant est trivial, mais il clarifie les choses.

Lemme 1.1. - Dans un sphère $S_{n}$, considérons trois droites $\delta_{1}, \delta_{2}$ et $\delta_{3}$ passant par le point $O$ et deux droites $D_{1}$ et $D_{2}$ immédiatement au-dessus de $\delta_{1}$ et $\delta_{2}$ respectivement. Alors il existe une droite $D_{3}$ au-dessus de $\delta_{3} q u i$ passe, tout comme $D_{1}$ et $D_{2}$ par $O^{\prime}$ au-dessus de $O$.

Démonstration. - Pensons au passage d'une sphère au link immédiatement supérieur. On se souvient qu'au-dessus d'un point, on place un plan affine et que les arêtes issues de ce sommet peuvent être vues comme les directions dans ce plan. Traduisons ce lemme dans ce langage. Considérons trois directions $\delta_{1}, \delta_{2}$ et $\delta_{3}$. Fixons-nous deux droites $D_{1}$ et $D_{2}$ dans les directions respectives $\delta_{1}$ et $\delta_{2}$. Alors, existe-t-il une droite $D_{3}$ dans la direction $\delta_{3}$, passant par le point d'intersection entre $D_{1}$ et $D_{2}$ ? La réponse est claire. $\square$ 
Considérons une configuration de Desargues $\left(A, B, C, A^{\prime}, B^{\prime}, C^{\prime}\right)$ de point de fuite $O$ (i.e. $O=\delta_{1} \cap \delta_{2} \cap \delta_{3}$ ). On peut relever $O$ en $O^{\prime}$ duquel partent trois brins rejoignant les droites au-dessus des $\delta_{i}$ (utiliser le lemme). Maintenant, relevons les $\delta_{i}$ en $D_{i}$ de telle sorte que les points $A, B, C, A^{\prime}, B^{\prime}, C^{\prime}$ se relèvent bien en des points passant par les $D_{i}$ correspondantes (on utilise ici encore le lemme).

Ainsi, d'étage en étage, on peut relever une configuration de Desargues générique. Cela montre qu'elle provient bien d'une configuration de Desargues de l'infini. Ici se termine notre éclaircissement.

Supposons donc construit un link d'ordre 2 (disons $\mathcal{L}$ ) au-dessus du link considéré. Relevons une configuration de Desargues générique. Si dans $\mathcal{L}$, cette configuration se complète symétriquement, il suffit de permuter les brins d'un bras (i.e. ensemble des droites parallèles du plan affine) du shadok dans lequel se trouve la droite de concours $\Delta$, pour qu'elle ne se complète plus symétriquement. Sur la figure 12, le point de concours des trois brins en traits épais représente cette droite $\Delta$. Nous avons choisi d'illustrer ce fait par une figure correspondant au cas $q=2$ pour bien montrer que ce n'est pas ce dernier point qui est mis en défaut dans le cas $q=2$, et bien sûr, pour ne pas compliquer les figures inutilement.

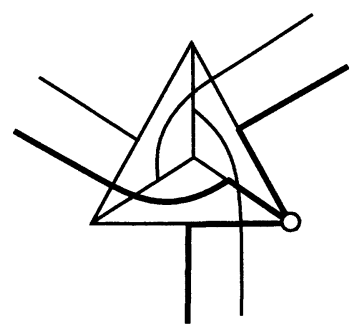

FIGURE 12

\section{Le $\operatorname{cas} q=2$}

Commençons par faire une remarque. Une configuration de Desargues dans le plan projectif $P^{2}\left(\mathbb{F}_{2}\right)$ est toujours dégénérée. En effet, si on considère deux triangles en perspective, l'un est nécessairement aplati. On peut facilement vérifier cela sur la figure schématique 13 où les sept droites sont matérialisées par six segments et un cercle (il ne faut pas confondre cette figure avec un shadok).

Il convient de noter que la construction du cas précédent n'est pas possible pour $q=2$. En effet, la droite d'alignement éventuel $\Delta$, fait partie de la configuration. Modifier ses branchements serait modifier la configuration 
initiale. Sur la figure 14, nous avons dessiné le graphe d'incidence d'une configuration de Desargues dans ce contexte. Les trois droites qui se trouvent sur le petit cercle central d'une configuration générique, sont ici confondues en une seule notée $D$.

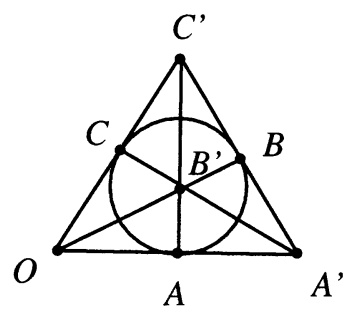

FIGURE 13

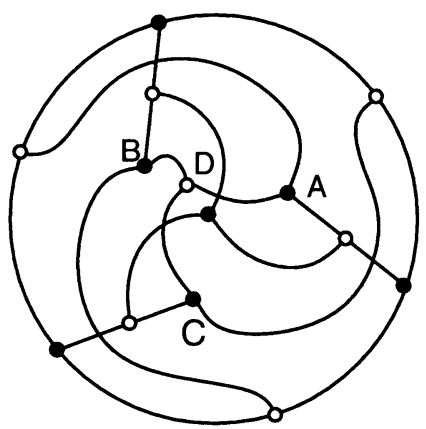

FIGURE 14

Il est à noter que ce graphe est le plan tout entier. Relevons la première partie de cette configuration (i.e. les deux points sur chacune des trois droites concourantes). On peut le faire, comme on l'a vu dans le cas précédent. Alors deux cas peuvent se produire (tout se passe au-dessus de la droite $D$ ):

- ou bien les points au-dessus de $A, B$ et $C$ sont alignés,

- ou bien les points d'intersection $(A B) \cap\left(A^{\prime} B^{\prime}\right),(A C) \cap\left(A^{\prime} C^{\prime}\right)$ et $(B C) \cap\left(B^{\prime} C^{\prime}\right)$ sont alignés.

Cette dichotomie se lit directement sur la figure 12 du paragraphe précédent (le shadok). Si trois droites viennent concourir dans ce shadok, les trois autres ne le font pas, et réciproquement. Quoiqu'il en soit, la propriété de Desargues est vérifiée. La droite en question dans cette propriété étant toujours celle dessinée sur la figure 12 .

Passons maintenant au link d'ordre trois. Partons de la configuration de Desargues de la sphère de rayon deux, qui correspond au second cas dans la 
dichotomie précédente. Il est désormais possible de construire un link d'ordre trois au-dessus d'une telle configuration qui nie la propriété de Desargues. En effet, tous les sommets sont alors différentiés et donc l'argument du cas $q \geqslant 3$ convient.

Afin de rassembler les résultats obtenus grâce à cette première étude de la tour des links, nous énoncons ici un théorème dont les assertions ont toutes été démontrées dans cette partie.

THÉORÈme 4. - - Pour tout $q$, il existe une infinité non dénombrable d'immeubles non deux à deux isométriques, ayant la même géométrie locale.

- Si $q \geqslant 5$, il existe des immeubles non réguliers.

- Pour $q \geqslant 3$ (resp. $q=2$ ), il existe des boules de rayon 2 (resp. 3) qui nient la propriété de Desargues.

On a vérifié à la main que l'immeuble exotique de la troisième partie de cet article, illustre le dernier point du théorème précédent : la propriété de Desargues est mise en défaut au niveau de la boule de rayon trois. L'idée qui ressort de ce dernier théorème est que plus la géométrie locale s'enrichit, plus le caractère non classique se localise.

\section{Prescription des boules de rayon 2}

Désormais, nous nous plaçons dans le cadre des immeubles triangulaires d'ordre 2. À la fin de cette partie, nous donnerons un exemple de prescription exotique. Pour ne pas alourdir le langage, on dira «une boule de rayon $n$ » pour « une boule de rayon combinatoire $n$ d'un certain immeuble triangulaire d'ordre 2.»

THÉORÈme 5. - Soient $B$ une boule de centre $O$ et de rayon $N$, et une application de prescription $p$ définie sur l'ensemble des sommets de la sphère $C$ de centre $O$ et de rayon $N-1$, à valeurs dans $\{0,1\}$. Il existe alors une boule $B^{\prime}$ de rayon $N+1$, de centre $O^{\prime}$ et une injection $j: B \longrightarrow B^{\prime}$ qui envoie $O$ sur $O^{\prime}$ et telles que pour tout sommet $S$ de $C$, la 2-boule de sommet $j(S)$ soit de type $p(S)$.

Démonstration. - Considérons une boule de rayon $N$. Nous commençons par la compléter de façon quelconque en une boule de rayon $N+1$. Nous allons maintenant modifier certaines parties de la sphère de rayon $N+1$ afin de satisfaire les contraintes imposées. Il s'agit de comprendre ce qui, dans la sphère de rayon $N+1$ contribue à la boule de rayon 2 d'un sommet $S$ de la sphère de rayon $N-1$. 


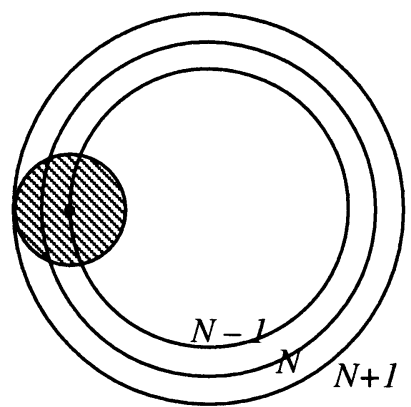

FIGURE 15

Nous distinguons deux cas suivant la nature du sommet $S$.

$1^{\text {er }}$ cas : $S$ est un sommet coin.

Dans $S_{N+1}$, quatre shadoks se trouvent au-dessus de $S$.

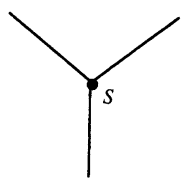

Niveau $N-1$

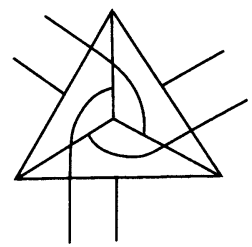

Niveau $N$

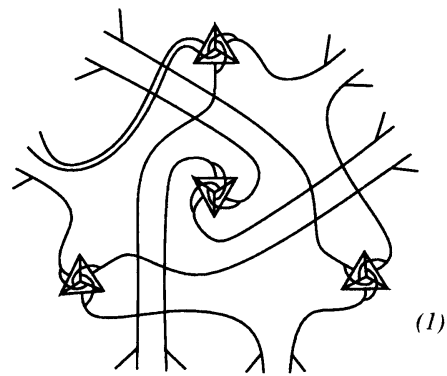

Niveau $N+1$

FIGURE 16

Le link d'ordre 2 du sommet $S$ contient exactement la partie (1) de la figure 16 comme sous-graphe. Attention, sur ce dessin, pour ne pas le surcharger, nous avons collé certaines portions d'arêtes. L'une de ces portions a été décollée sur la figure pour montrer de quoi il s'agit (au Nord-Ouest). Nous décidons d'appeler vrilles associées les douze petits brins (ou fourches) issus de ce "gros shadok». Remarquons que modifier l'une de ces vrilles associées sans rien modifier ailleurs revient à changer le type du link d'ordre 2 de $S$.

$2^{e m e}$ cas : $S$ est un sommet non coin.

Dans $S_{N+1}$, huit octogones se trouvent au-dessus de $S$. 


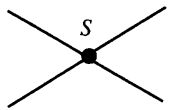

Niveau $N-1$

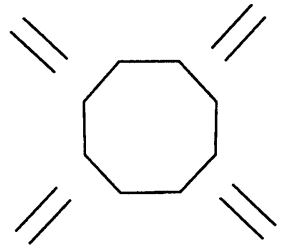

Niveau $N$

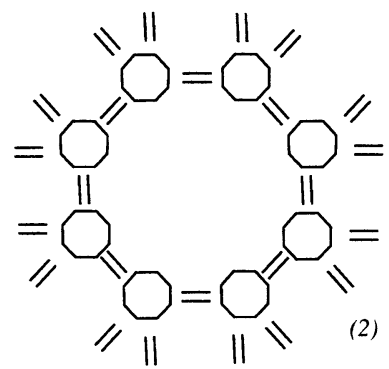

Niveau $N+I$

FigURE 17

Le link d'ordre 2 du sommet $S$ contient exactement la partie (2) de la figure 17 comme sous-graphe. Nous décidons d'appeler vrilles associées les huit petits brins formant l'ossature du «gros octogone». Remarquons ici encore que modifier l'une de ces vrilles associées sans rien modifier ailleurs revient à changer le type du link d'ordre 2 de $S$.

Pour conclure quant à l'existence d'un immeuble à boules de rayon 2 prescrites, il nous faut faire la remarque fondamentale suivante :

Affirmation 1.- Modifier l'une des vrilles associées au sommet $S$ change la sphère de rayon 2 de ce sommet sans modifier les sphères de rayon 2 des autres points de $S_{N-1}$.

Démonstration. - Nous avons déjà vu que la 2-sphère de $S$ était modifiée. Voyons maintenant pourquoi les autres ne le sont pas. Regardons comment interviennent les vrilles associées à un sommet $A$ dans la boule de rayon 2 d'un autre sommet $B$. Pour cela, sur la figure 18 on représente les niveaux $N$ et $N+1$ dans un plat. On note $a$ et $b$ les secteurs d'un triangle choisi, intervenant dans le link d'ordre 2 de $A$ et $B$ respectivement.

Sur la partie inférieure de la figure 18, on montre l'effet de la vrille au niveau de $a$ dans le link d'ordre 2 en $A$ sur le link d'ordre 2 en $B$. On voit donc que le link d'ordre 2 en $B$ ne se trouve pas modifié par une vrille associée à $A$. La situation que nous venons de décrire est générale. En effet, si le sommet $B$ se trouve à distance $\geqslant 2$ de $A$, son link d'ordre 2 ne fait pas intervenir les vrilles associées à $A$; par exemple, sur la figure 19, le triangle grisé n'intervient pas dans la boule de rayon 2 , de centre $B$.

Remarque sur l'unicité à prescription donnée. - Parmi les immeubles classiques, il en existe une infinité qui ont les mêmes boules de rayon 2 (c'est encore vrai pour les boules de rayon $\mathrm{n}$ ). Il en est de même pour les 
immeubles exotiques (on peut même ajouter que cette infinité n'est pas dénombrable). Plus précisément, on peut construire par exemple une boule de rayon 4 dont les links d'ordre 2 sont prescrits de façon quelconque, et qui ne vérifie pas la propriété de Desargues.

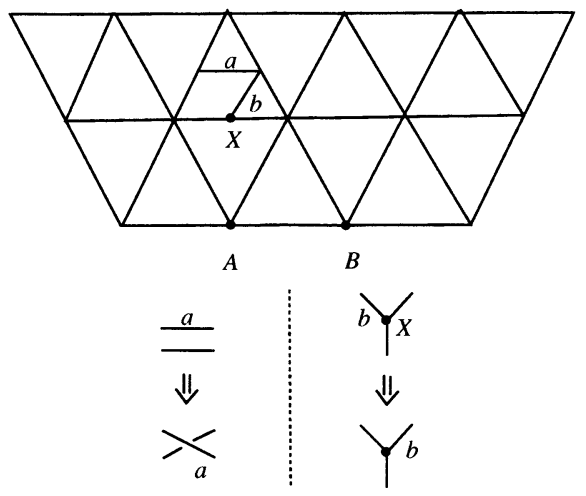

Figure 18

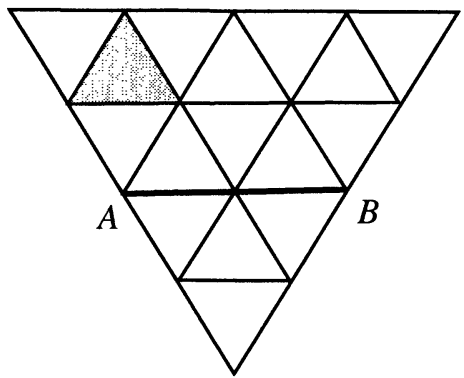

FiguRE 19

Donnons maintenant un exemple de prescription exotique sur les sommets. À l'aide du tricoloriage classique des sommets des immeubles euclidiens de dimension 2, construisons un immeuble triangulaire d'ordre 2 dont les boules de rayon 2 sont prescrites de la façon suivante. Si $A, B$ et $C$ sont les trois couleurs, demandons que les boules de rayon 2 des sommets $A$ soient d'un type et que celles des sommets $B$ ou $C$ soient de l'autre. Ainsi, l'ensemble des sommets se verra bicolorié par le type des boules de rayon deux de telle sorte que dans chacun des appartements, on voit se dessiner le pavage hexagonal. Voir la figure 20. 


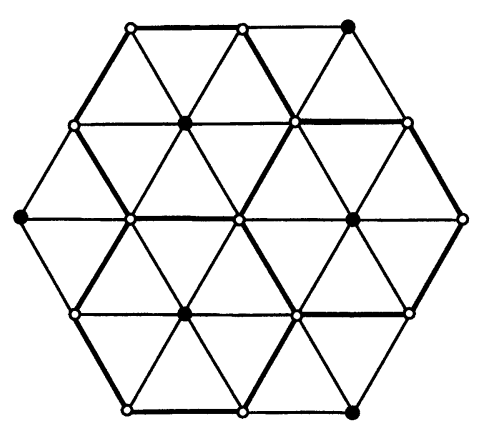

FIGURE 20

Un sommet noir (de type $A$ ) se trouve bordé de sommets blancs (type $B$ ou $C$ ), et un sommet blanc, de sommets noirs et blancs alternés. Un tel immeuble possède «deux fois plus» de sommets blancs que de sommets noirs. Aussi, certains d'entre eux sont probablement très homogènes. Ces deux remarques nous suggèrent l'existence de polyèdres à trois sommets (deux blancs et un noir) dont le revêtement universel soit un de ces immeubles exotiques (où on a convenu d'appeler polyèdre un quotient d'un immeuble euclidien par un groupe qui opère librement et de façon co-compacte). Je pense qu'il serait intéressant de reprendre les exemples de Ronan [R2] en suivant cette idée là. Avant de passer à trois sommets, nous voulions poursuivre nos recherches sur des quotients à deux sommets, nous avons découvert alors le polyèdre extraordinaire décrit dans la partie suivante.

\section{Un polyèdre exotique}

Un polyèdre triangulaire d'ordre 2 à deux sommets possède, a priori, quatre types de faces représentées sur la figure 21 .

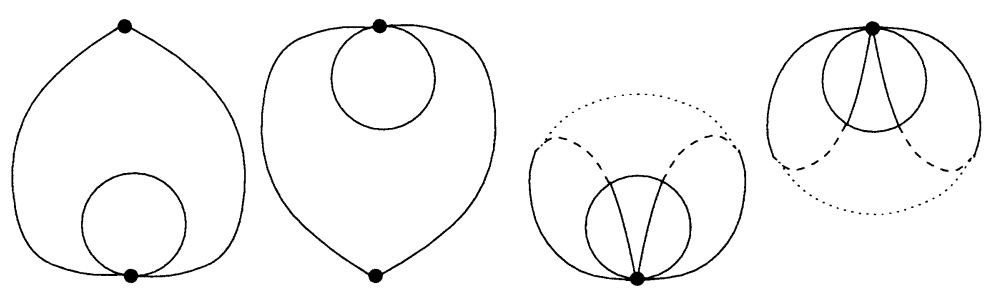

FIGURE 21

Après avoir enlevé des boules de rayon $\varepsilon$ centrées sur chacun des sommets, des faces il ne reste que des hexagones (nous avons tracé un de ces hexagones sur la figure 22). 
Les arêtes notées $a_{i}$ composent les sphères de rayon $\varepsilon$ (ou links en les sommets), tandis que les autres notées par des entiers, seront tracés sur la figure 23 reliant les sommets des deux links. Nous obtenons ainsi une représentation complète et assez explicite de notre polyèdre $P$. On conviendra que l'un des sommets est noir et que l'autre est blanc. Les faces notées $a, b, c, d, e, f$ possèdent au moins deux sommets blancs ; les autres, notées $a^{\prime}, b^{\prime}, c^{\prime}, d^{\prime}, e^{\prime}, f^{\prime}, g^{\prime}, h^{\prime}$, au moins deux noirs. Notre polyèdre $P$ est donc entièrement défini par la figure 23 . Le sommet blanc y est représenté au-dessus du noir.

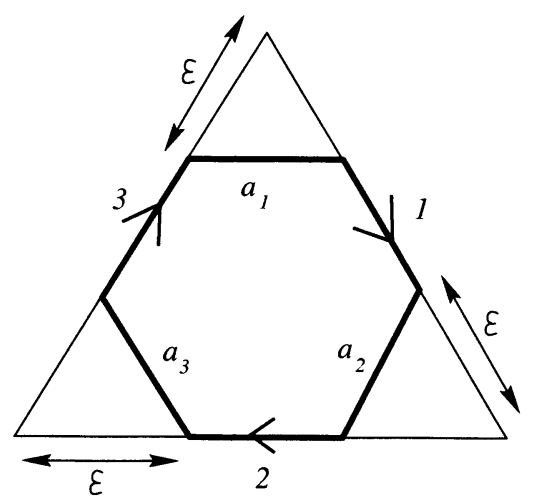

Figure 22

THÉORÈME 6. - Dans le revêtement universel de $P$, la boule de rayon 2 du sommet noir n'est pas isomorphe à celle du sommet blanc.

Démonstration. - Un calcul manuel des 2-boules de chacun des sommets (voir [Ba2] pour plus de détails) nous donne ce résultat. Plus précisément, la 2-boule du sommet blanc (resp. noir) est celle de l'immeuble associé à $\mathbb{F}_{2}((t))$ (resp. $\left.\mathbb{Q}_{2}\right)$. Le sommet noir est de type 1 tandis que le blanc est de type 0 (voir la figure 3 ).

Les sommets du revêtement universel $\widetilde{P}$, sont donc naturellement colorés suivant le type de leur 2-boules. Aussi, puisque le link en un sommet peut être vu comme la sphère de rayon un, ses sommets, qui sont alors aussi des sommets de $\widetilde{P}$, se trouvent colorés. Les links colorés en un sommet noir et en un sommet blanc sont représentés sur la figure 24 . 
Sylvain Barré

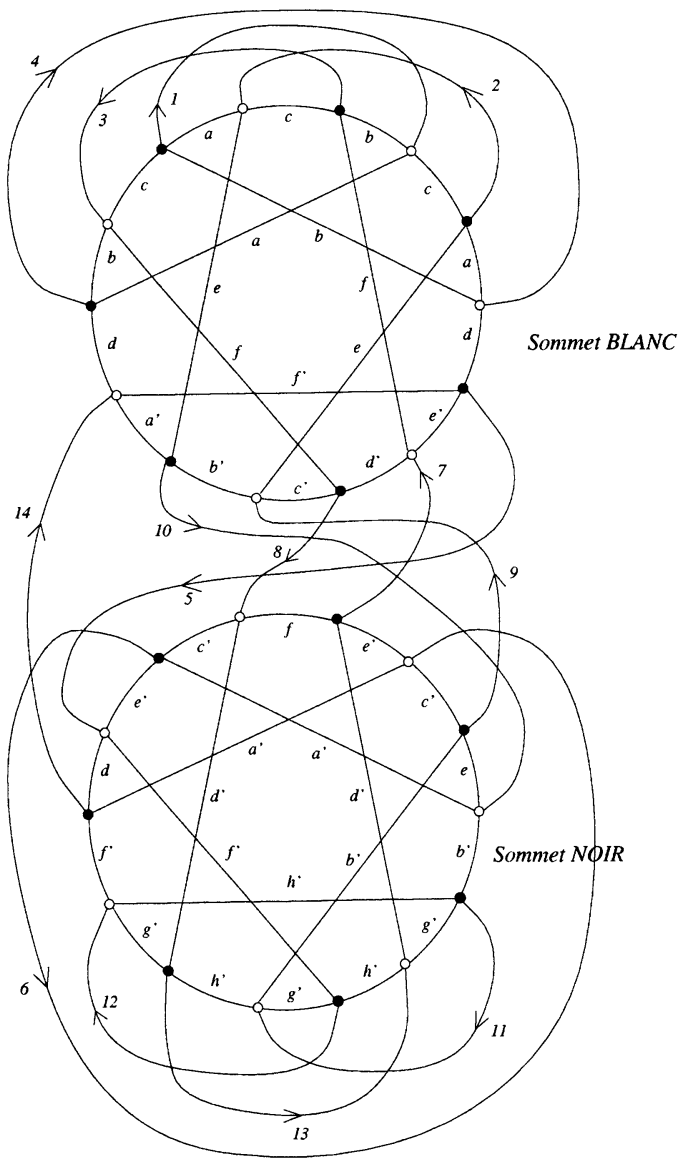

FIGURE 23

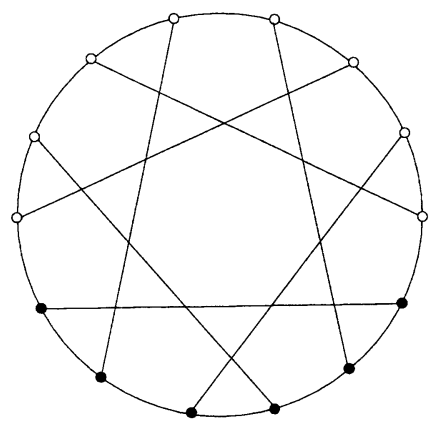

Sommet BLANC

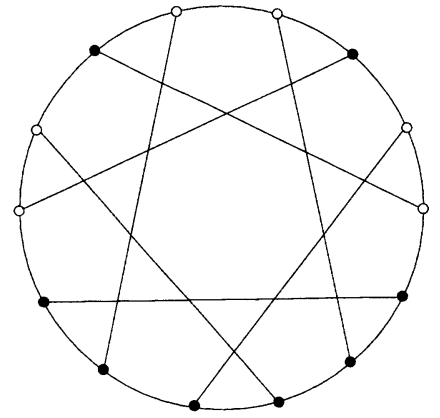

Sommet NOIR

Figure 24 
Avant d'énoncer le théorème principal dont la clé se trouve dans le théorème précédent, indroduisons un autre élément. On appellera plan médian, l'ensemble des points équidistants des deux sommets. Il s'agit d'un graphe dont les sommets correspondent aux arêtes reliant les deux sommets du polyèdre. Le plan médian de $P$ est dessiné sur la figure 25 . Les arêtes sont de deux types suivant qu'elles correspondent à une arête du link du sommet noir (arêtes en gras) ou du sommet blanc (arêtes fines).

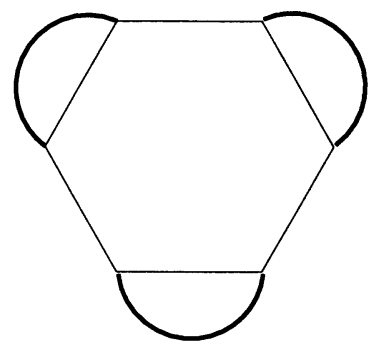

FIGURE 25

THÉORÈme 7. - Le groupe fondamental $\pi_{1}(P)$ est d'indice fini ( 6 ou 12) dans le groupe des automorphismes de $\widetilde{P}$.

Démonstration. - Démontrons pour commencer qu'il est d'indice au plus 12. Nous verrons ensuite, en étudiant les isométries de $P$ que cet indice est au moins 6 . Bien sûr, $A u t(\widetilde{P})$ préserve le bicoloriage des sommets. Considérons un élément $g \in \operatorname{Aut}(\widetilde{P})$. Quitte à le composer avec un élément de $\pi_{1}(P)$, on peut supposer que $g$ fixe un sommet noir $Q_{0}$. Avant de détacher un petit lemme, fixons nous un peu de vocabulaire. Étant donné, dans un link, un petit cycle (de longueur six), il existe exactement deux sommets de ce link à distance 2 de ce cycle. Ces points seront appelés points orthogonaux au cycle. Aussi, on dira que les trois arêtes issues d'un sommet orthogonal forment un tripode orthogonal.

Lemme 3.1. - Soit $\tau$ un automorphisme du link. Si l'une des deux conditions suivantes est vérifiée, alors $\tau$ est l'identité.

1. $\tau$ fixe un petit cycle point par point.

2. $\tau$ fixe globalement un petit cycle et fixe de plus un tripode orthogonal point par point.

Démonstration. - Un automorphisme vérifiant 1. est une transformation projective de $P^{2}\left(\mathbb{F}_{2}\right)$ qui fixe les trois vecteurs d'une base de $\mathbb{F}_{2}^{3}$. Puisqu'il n'y a pas de scalaire non trivial dans $\mathbb{F}_{2}$, une telle transformation est triviale. 
Un automorphisme qui vérifie 2 . fixe aussi le deuxième point orthogonal car l'ensemble des deux points orthogonaux doit être globalement fixe comme le petit cycle. Or les trois sommets terminaux du tripode fixe avec le second sommet orthogonal constituent les quatre points d'une base projective. Ainsi l'automorphisme en question est trivial.

À indice 12 près, supposons que $g$ fixe point par point le cycle noir du link en $Q_{0}$ (on savait qu'il le fixait globalement). D'après le lemme, $g$ fixe sa boule de rayon un point par point. Notons $B\left(Q_{0}, N\right)$ (resp. $S\left(Q_{0}, N\right)$ ) la boule (resp. la sphère) de rayon $N$ centrées en $Q_{0}$. Regardons maintenant comment l'automorphisme $g$ opère autour des sommets noirs de $S\left(Q_{0}, 1\right)$. Pour les six qui composent le petit cycle en $Q_{0}$, on sait que deux arêtes consécutives du petit cycle noir de leur link sont fixées. Pour les deux autres, on sait qu'un tripode orthogonal au petit cycle noir de leur link est fixe. Ainsi, toujours d'après le lemme, toutes les boules $B\left(Q_{1}, 1\right)$ sont fixes point par point où $Q_{1}$ est un sommet noir de $S\left(Q_{0}, 1\right)$. Considérons maintenant un sommet blanc de $S\left(Q_{0}, 1\right)$. On sait qu'il y a alors deux arêtes consécutives dans le petit cycle noir de son link, qui son fixes. Le lemme nous dit alors que toute sa boule est fixe.

Pour résumer, nous avons montré que si $g$ fixe la boule de rayon un centrée sur un sommet noir, il fixe aussi la boule de rayon deux, point par point. Appelons $\left(^{*}\right)$ cette propriété.

Remarquons maintenant la propriété fondamentale de cet immeuble $\widetilde{P}$.

PropriÉté 1. - L'ensemble des sommets noirs s'organise dans $\widetilde{P}$ en une union de plats deux à deux disjoints.

Démonstration. - On dira qu'une face est noire si ses trois sommets sont noirs. Considérons un sommet noir. Il a 6 voisins noirs avec qui il forme un hexagone noir $H$ (c'est-à-dire un hexagone composé de 6 triangles n'ayant que des sommets noirs). Ses deux autres voisins noirs sont les points orthogonaux au cyle noir). Les hexagones noirs de chacun des sommets de $H$ viennent border $H$ pour former un hexagone de rayon 2. Ainsi, l'ensemble des sommets noirs est contenu dans une union de plats noirs. Deux plats noirs sont disjoints puisque le cycle noir dans le link d'un sommet noir est à distance 2 des deux autres sommets noirs de ce link.

Regardons maintenant le 1-squelette de $\widetilde{P}$. Notons $N$ le sous-graphe composé des arêtes reliant sommet noir à sommet noir. Démontrons que $N$ est connexe. Considérons une composante connexe $N_{0}$ de $N$, et deux sommets noirs $A$ et $B$ qui réalisent la distance combinatoire $d$ entre $N_{0}$ et son complémentaire $N-N_{0}$. Il est clair que $d \leqslant 3$ car un sommet blanc a des 
voisins noirs. Puisque $A$ et $B$ se trouvent dans un même plat de $\widetilde{P}$, il y a un chemin géodésique les reliant, composé d'arêtes et formant éventuellement un angle de $2 \pi / 3$ sur son parcours. La distance $d$ ne peut pas être égale à 2 , car le cycle noir du link du sommet blanc intermédiaire donnerait une connexion entre $A$ et $B$. Supposons donc $d=3$. Notons $X$ et $Y$ les deux sommets intermédiaires de $A$ à $B$ (voir figure 26). Les trois sommets à distance un de $X$ et de $Y$ dans $\widetilde{P}$ sont blancs. Notons $Z$ l'un d'eux.

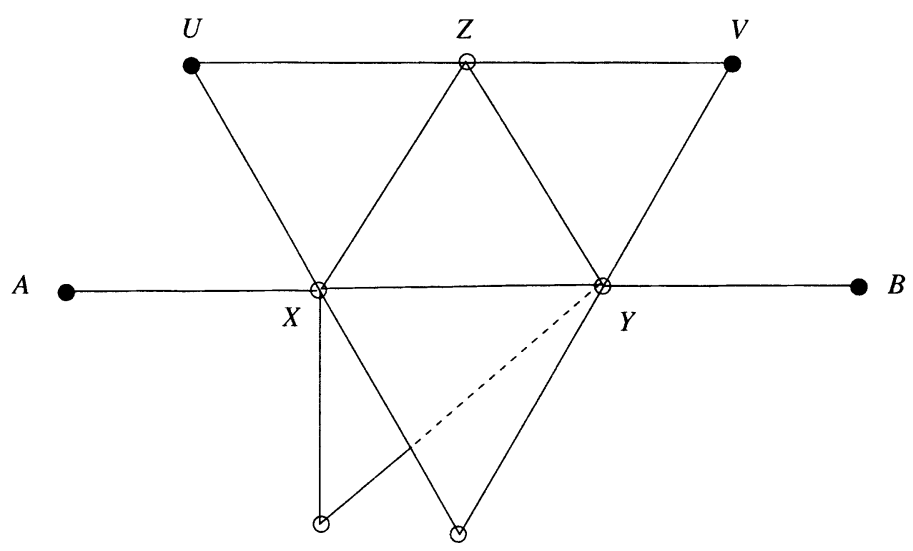

FIGURE 26

En regardant le link en $X$, compte tenu de la position du tipode blanc, on s'aperçoit que $X$ (resp. $Y$ ) et $Z$ ont un sommet noir commun à distance un noté $U$ (resp. $V$ ). Mais alors les trois distances $d(A, U), d(U, V)$ et $d(V, B)$ sont $\leqslant 2$, ce qui n'est pas possible on l'a vu. L'ensemble noir $N$ est donc connexe.

La propriété $\left.{ }^{*}\right)$ et la connexité de $N$ implique que $g$ laisse fixes tous les sommets noirs et leur voisinage. Ainsi $g$ est l'identité. Nous avons donc démontré que l'indice de $\pi_{1}(P)$ est au plus 12 dans le groupe d'automorphismes de $\widetilde{P}$. Pour voir qu'il est au moins de 6 , cherchons les isométries de $P$.

AfFirmation. - Toute symétrie du plan médian se relève en une isométrie de $P$ et de façon unique.

La démonstration de cette affirmation se fait directement sur la représentation donnée de $P$. Les symétries apparaissent nettement. L'unicité du relèvement est claire compte tenu du lemme précédent.

Pour finir, nous donnons une présentation de $\pi_{1}(P)$ assez symétrique avec quatre générateurs : $a, b, c$ et $d$ correspondant respectivement aux arêtes 
numérotées $11,12,13$ et 6 sur la figure 23 . On note $[x, y]$ le commutateur $x y x^{-1} y^{-1}$.

$$
\begin{gathered}
\pi_{1}(P)=<a, b, c, d \quad \mid \quad a b c=1 \quad, \quad[a, b]=1 \\
{[d, a]\left[b^{-1}, d^{-1}\right]=d^{3},} \\
\left(d^{-1} c^{-1} d\right) a\left(d^{-1} c d\right)=a\left(d^{-1} c d\right) a^{-1}=a^{-1}\left(d b d^{-1}\right) a \quad>
\end{gathered}
$$

Cette présentation laisse apparaître deux choses :

- d'une part que $a, b$ et $c$ sont conjugués,

- d'autre part que $d^{3}$ est le produit de deux commutateurs.

De la première affirmation, on tire géométriquement que $a^{3 n}$ (et bien sûr $b^{3 n}$ et $c^{3 n}$ ) sont le produit de seulement deux commutateurs, quel que soit $n$. D'autre part, l'action de $d^{3}$ sur $X$ est très similaire à celle de $a^{3}$ (nous pensons que ces éléments sont conjugués). Ainsi, nous conjecturons que tout élément du groupe dérivé de $\pi_{1}(P)$ (qui est d'indice $3 \times 3$ dans $\pi_{1}(P)$ ) est le produit de seulement deux commutateurs.

\section{Bibliographie}

[A] ARTin (E.). - Algèbre géométrique, Bordas, Paris, 1978.

[Ba1] BARRÉ (S.). - Polyèdres finis de dimension 2 à courbure négative ou nulle et de rang 2, Annales de l'Institut Fourier, T45, 1995.

[Ba2] BARRÉ (S.). - Polyèdre de rang deux, thèse, ENS Lyon, décembre 1996.

[BB] Ballmann (W.), Brin (M.). - Orbihedra of nonpositive curvature, Publications Mathématiques de l'IHES, 82, 1995.

[Bro1] Brown (K.S.). - Buildings, Springer-Verlag, New York 1989.

[Bro2] Brown (K.S.). - Five lectures on buildings, Congrès de Trieste 1990.

[BH] BRIDSON (M.), HAEFLiger (A.). - Metric spaces of non-positive curvature, Grundlehren der Mathematischen Wissenschaften, 319, Berlin 1999.

[CMSZ] Cartwright (D.I.), Mantero (A.M.), Steger (T.), Zappa (A.). - Groups acting simply transitively on the vertices of a building of type $\widetilde{A}_{2} I$; The cases $q=2$ and $q=3$, II Geom. Dedicata 47 (1993), $\mathrm{n}^{\circ} 2,143-166-223$.

[CMS] Cartwright (D.I.), Mlotkowski (C.), Steger (T.). - Property (T) and $\widetilde{A}_{2}$ groups, Ann. Inst. Fourier 44 (1994), $\mathrm{n}^{\circ}$ 1, 213-248.

[R1] Ronan (M.A.). - Lectures on Buildings, Perspectives in Mathematics, Vol. 7, Academic Press 1989.

[R2] RONAN (M.A.). - A universal construction of buildings with no rank 3 residue of spherical type, édité par L. A. Rosati, Buildings and the geometry of diagrams, Proceedings Como 1984, Lectures Notes in Mathematics 1181 (Springer-Verlag, 1986), 242-248.

[R3] Ronan (M.A.). - Triangle Geometries, Journal of Combinatorial Theory, Series A 37, 294-319 (1984). 
Immeubles de Tits triangulaires exotiques

[RT] Ronan (M.A.), Tits (J.). - Building Buildings, Math. Ann. 278, (1987) 291306.

[T1] TITs (J.). - Spheres of Radius 2 in Triangle Buildings. I. Finite geometries buildings and related Topics. Pingrie Park Conference, 1988.

[T2] TITs (J.). - A local approach to Buildings, The Geometric Vein (Coxeter Festschrift), 519-547. Berlin, Heidelberg, New York ; Springer 1981

[T3] TITs (J.). - On buildings and their Applications, Proceedings of the International Congress of Mathematicians ; Vancouver, 1974.

[T4] Tits (J.). - Immeubles de type affine, édité par L. A. Rosati, Buildings and the geometry of diagrams, Proceedings Como 1984, Lectures Notes in Mathematics 1181 (Springer-Verlag, 1986), 157-190.

[T5] TITs (J.). - Lecture Notes in Mathematics 386, Springer Verlag 1974.

[TM] TITS (J.), MÜLLER. - Livre en préparation.

[VM1] VAN Maldeghem (H.). - Non-classical triangle buildings, Geom. Dedicata 26 (1988), 29-84.

[VM2] VAn Maldeghem (H.).- - On projective Hjelmslev-planes of level n, Glasgow Math. J.31, (1989), 257-261.

[VMVS1] VAn Maldeghem (H.), VAn Steen. - Moufang affine buildings have Moufang spherical buildings at infinity, Glasgow Math. J. 39, (1997), 237-241.

[VMVS2] VAN Maldeghem (H.), VAN SteEn. - Characterizations by automorphism groups of some rank 3 building. I : Some properties of half stongly-transitive triangle buildings. Geom. Dedicata 73, No. 2, 119-142 (1998).

[VMVS3] VAN Maldeghem (H.), VAN Steen. - Characterizations by automorphism groups of some rank 3 building. II : A half stongly-transitive locally finite triangle building is a Bruhat-Tits building. Geom. Dedicata 74, No. 2, 113133 (1999).

[VS] VAN STEEN. - Characterizations by automorphism groups of some rank 3 building. III : Moufang-like conditions. Geom. Dedicata 74, No. 3, 225-240. 